\title{
Structural modal reanalysis of topological modifications
}

\author{
Su Huan Chen ${ }^{\mathrm{a}, *}$, Cheng Huang ${ }^{\mathrm{a}}$ and \\ Zongjie Cao ${ }^{\mathrm{b}}$

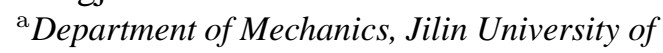 \\ Technology, Changchun 130022, People's Republic of \\ China \\ ${ }^{\mathrm{b}}$ The Second Aeronautic Institute of Air Force, \\ Changchun 130022, People's Republic of China
}

Received 2 March 1999

Revised 30 November 1999

This paper presents a procedure for modal reanalysis of structures with topological modifications. The procedure is based on the results of initial modal analysis of the original structure. It is necessary for topological modifications with the increase of joints and the number of degrees of freedom. The research results show that the proposed method is effective and easy to implement on a computer.

Keywords: Topological modification, structural modal reanalysis, finite element system

\section{Introduction}

The modifications of structures and the resolution of general eigenproblems are the main problems dealt with in engineering fields to achieve an optimal design. The iterative vibration analysis can be expensive for large and complex structures. Therefore it is necessary to seek a faster computation method for reanalysis. Various modifications in the structural design are classified into three types according to the modified patterns: parameter, shape, and topology. Parameter modifications involve modifying structural parameters, such as cross-sectional area, mass and material elastic rigidity, etc., with boundary shapes and topology unchanged, which are referred to in $[1,2,4,6,10]$. The methods for parameter modifications can not be applied to the case of the topological modifications. Shape modifications

\footnotetext{
${ }^{*}$ Corresponding author.
}

involving boundary modifications with the topology of structures fixed are presented in [5,11]. Topological modifications concerning changes of topology of a structure (number and orientation of elements) are more difficult because they involve the changes in the structural model. More efforts are still required to implement topological modifications in practical structural designs [8].

Possible modifications in topological optimizations can be classified as follows [7]:

1) Deletion of members and joints, where both the design variable vector and the number of DOF are reduced. When only members are deleted, the values of some design variables become zero and can be eliminated from the set of variables.

2) Addition of members and joints, where both the design variables and the number of DOF are increased. When members are added without addition of joints, the vector of design variables is expanded, but the number of DOF is unchanged.

3) Modification in geometry, where there is no change in the number of variables and DOF. In this case, only the numerical values of variables are modified.

In previous studies, significant progress has been achieved. The static reanalysis procedures for all the above-mentioned cases of layout modifications are developed in [3,9]. However, many methods for modal reanalysis of parameter modifications of structures, such as Ritz analysis and matrix perturbation, can not be directly used to deal with the modal analysis for layout modifications in which the number of DOF will be increased (case 2).

This study presents a new, simple and convenient procedure for introducing expanded basis vectors. By this approach, the stiffness matrix and mass matrix of modified structures are formed directly with the submatrices of augmented stiffness matrix and mass matrix. The procedure is suitable for the changes in a general finite element system. Once expanded, basis vectors are formed, and the one-step subspace iteration is used 
to obtain an approximate result of the modified structure. The calculations are based on results of initial modal analysis. Each step of the subsequent reanalysis involves the solution of a small-order system of equations. Therefore, the computational efforts can be significantly reduced.

\section{Technical background}

The most important of general techniques for finding approximations to the lowest eigenvalues and corresponding eigenvectors of the problem $K \Phi=\lambda M \Phi$ is the Rayleigh-Ritz analysis. Various methods can be considered as Ritz analysis. The techniques differ only in the choice of Ritz basis vectors assumed in the analysis.

Since there are only minor modifications in the dynamic optimization, the eigensystem of the previous structure can be a good approximation to the eigensystem of the new structure. The eigenvectors $\Phi_{0}$ of initial structure can be used as Ritz basis vectors, where $\Phi_{0}$ is an $n \times q$ matrix, i.e., $\Phi_{0}=\left[\phi_{01}, \cdots, \phi_{0 q}\right]$. The analysis can be continued by evaluating the projections of $K$ and $M$ onto the subspace $V_{q}$ spanned by the vectors $\phi_{0 i}, i=1, \cdots, q$; i.e., where

$$
\bar{K}=\Phi_{0}^{T} K \Phi_{0}
$$

and

$$
\bar{M}=\Phi_{0}^{T} M \Phi_{0}
$$

where $q \ll n$.

Next step is to solve the eigenproblem,

$$
\bar{K} X=\bar{M} X \rho
$$

where $\rho$ is a diagonal matrix listing the eigenvalue approximations $\rho_{i}, \rho=\operatorname{diag}\left(\rho_{i}\right)$, while $X$ is a matrix storing the $\bar{M}$-orthonormal eigenvectors $x_{i}, \cdots x_{q}$, the approximations to the eigenvectors of the problem, $K \Phi=\lambda M \Phi$, are as follows:

$$
\Phi=\Phi_{0} X
$$

After the Ritz analysis, the original large-order system can be reduced to a small-order one.

\section{Problem formulation}

Various changes of the structure will result in changes of the stiffness matrix $K$ and the mass matrix $M$. The generalized eigenproblem of the modified structure is

$$
\begin{aligned}
K_{m} \Phi & =\left(K_{0}+\Delta K\right) \Phi=\lambda M_{m} \Phi \\
& =\lambda\left(M_{0}+\Delta M\right) \Phi
\end{aligned}
$$

where $K_{0}$ and $M_{0}$ are the stiffness matrix and mass matrix of the initial structure; $K_{m}$ and $M_{m}$ are modified stiffness matrix and mass matrix; $\Delta K$ and $\Delta M$ are the changes in the stiffness matrix and mass matrix respectively.

In the structural optimization, topological modification involving changes in members and joints is commonly divided into three cases with the change of DOF:

1) The common case, where the number of DOF is unchanged;

2) The case, where the number of DOF is decreased;

3) The most challenging case, where the number of DOF is increased.

Most of the reanalysis methods developed in the past are suitable for cases 1) and 2). The reanalysis method developed in this study is to include problems where the number of DOF is increased.

\subsection{Case I (number of DOF is unchanged)}

Assuming the change in the design variables is denoted by $\Delta X$, the corresponding design variables of the modified design can be expressed as

$$
X=X_{0}+\Delta X
$$

and the corresponding stiffness matrix and mass matrix are given by

$$
K=K_{0}+\Delta K, M=M_{0}+\Delta M
$$

where $\Delta K$ and $\Delta M$ are the changes of $K_{0}$ and $M_{0}$ due to the change of $\Delta X$. The elements of the stiffness matrix and mass matrix are not restricted to certain forms and can be general functions of the design variables, that is, the design variables $X$ can be the function of coordinates of joints, the structural shape, cross sections of members, etc.

\subsection{Case II (number of DOF is decreased)}

Consider the case where some joints and some members connecting these joints to remaining joints are eliminated from the initial structure so that the number of DOF can be decreased. Assuming that the eliminated DOF are the last numbers of DOF, then $\Delta K$ and $\Delta M$ in Eq. (5) can be divided into submatrices as follows: 


$$
\begin{gathered}
\Delta K=\left[\begin{array}{ll}
\Delta K_{r r} & \Delta K_{r d} \\
\Delta K_{d r} & \Delta K_{d d}
\end{array}\right], \\
\Delta M=\left[\begin{array}{ll}
\Delta M_{r r} & \Delta M_{r d} \\
\Delta M_{d r} & \Delta M_{d d}
\end{array}\right]
\end{gathered}
$$

where $\Delta K_{d d}$ and $\Delta M_{d d}$ are the submatrices of stiffness and mass coefficients of the eliminated joints, in which subscript $d$ denotes the number of the deleted DOF, and $r$ the number of DOF of the remained structure.

\subsection{Case III (number of DOF is increased)}

Consider the case where new joints and some members connecting these joints to existing joints are added to the initial structure so that the number of DOF can be increased.

The $\Delta K$ and $\Delta M$ in Eq. (5) can be partitioned into

$$
\begin{gathered}
\Delta K=\left[\begin{array}{ll}
\Delta K_{n n} & \Delta K_{n m} \\
\Delta K_{m n} & \Delta K_{m m}
\end{array}\right], \\
\Delta M=\left[\begin{array}{ll}
\Delta M_{n n} & \Delta M_{n m} \\
\Delta M_{m n} & \Delta M_{m m}
\end{array}\right]
\end{gathered}
$$

where $\Delta K_{m m}$ and $\Delta M_{m m}$ are the submatrices of stiffness and mass coefficients of the new added joints, in which subscript $n$ denotes the number of DOF of the initial structure, and $m$ the augment of DOF of the modified structure, thus obtaining

$$
K_{m}=\left[\begin{array}{cc}
K_{0} & 0 \\
0 & 0
\end{array}\right]+\left[\begin{array}{ll}
\Delta K_{n n} & \Delta K_{n m} \\
\Delta K_{m n} & \Delta K_{m m}
\end{array}\right]
$$

and

$$
M_{m}=\left[\begin{array}{cc}
M_{0} & 0 \\
0 & 0
\end{array}\right]+\left[\begin{array}{ll}
\Delta M_{n n} & \Delta M_{n m} \\
\Delta M_{m n} & \Delta M_{m m}
\end{array}\right]
$$

For the lumped mass matrix, $\Delta M_{n m}$ and $\Delta M_{m n}$ are equal to zero.

The problem under consideration can be formulated as follows:

Given $K_{0}, M_{0}$ and eigenpairs $\left(\Phi_{0}, \lambda_{0}\right)$, the goal is to find efficient and high-quality approximations of the modified eigenpairs $\left(\phi_{i}, \lambda_{i}\right)$ that result from various changes in the structure without resolving Eq. (5) of the structure modified. To this end, a new approach for the modal analysis of structural layout modifications is presented in the next section. For completeness of presentation, the approach for the first two cases is first described and then the method for the case where the number of DOF is increased is developed.

\section{Reanalysis method}

\subsection{Case I and II (number of DOF is not increased)}

The Rayleigh-Ritz analysis in different forms has been used to deal with problems with unchanged numbers of design variables and DOF. As demonstrated in Eqs. (1) to (4), the eigenvectors of initial structure can be used as the Ritz basis vectors. In the case where the number of DOF is decreased, the corresponding DOF in the eigenvectors can be eliminated to get the Ritz basis vectors.

\subsection{Case III (number of DOF is increased)}

\subsubsection{Expanding the basis vectors}

If some joints are added to the structure, thus increasing the number of DOF and expanding the size of stiffness matrix and mass matrix, it is necessary first to expand the basis vectors, so that the new DOF are included in the new analysis model.

Given initial design and the addition of new joints and members, the changes in the stiffness matrix and mass matrix can be partitioned as in Eq. (9). The vibration eigenproblem of the modified structure is

$$
\begin{aligned}
& {\left[\begin{array}{cc}
K_{0}+\Delta K_{n n} & \Delta K_{n m} \\
\Delta K_{m n} & \Delta K_{m m}
\end{array}\right] \Phi_{m}} \\
& =\left[\begin{array}{cc}
M_{0}+\Delta M_{n n} & \Delta M_{n m} \\
\Delta M_{m n} & \Delta M_{m m}
\end{array}\right] \Phi_{m} \Lambda_{m}
\end{aligned}
$$

It is unnecessary to reassemble the whole stiffness matrix and mass matrix of the modified structure, which only needs to assemble the changes of stiffness matrix and mass matrix including the added new nodes and members.

The exact results can be obtained from Eq. (12). However, with the results from the initail analysis the approximate solutions can be obtained without resolving Eq. (12). Assuming that the approximate expressions for $\Phi_{m}$ and $\Lambda_{m}$ are as follows:

$$
\Phi_{m}=\left\{\begin{array}{c}
\Phi_{0} \\
\Delta \Phi
\end{array}\right\}
$$

and

$$
\Lambda_{m}=\Lambda_{0}
$$

Substituting Eqs. (13) and (14) into (12) and expanding the second equation of Eq. (12), the following equation can be obtained:

$$
\begin{aligned}
& \left(\Delta K_{m n}-\lambda_{0 i} \Delta M_{m n}\right) \phi_{0 i} \\
& =-\left(\Delta K_{m m}-\lambda_{0 i} \Delta M_{m m}\right) \Delta \phi_{i}
\end{aligned}
$$


From the above equation,

$$
\begin{gathered}
\Delta \phi_{i}=-\left(\Delta K_{m m}-\lambda_{0 i} \Delta M_{m m}\right)^{-1} \\
\left(\Delta K_{m n}-\lambda_{0 i} \Delta M_{m n}\right) \phi_{0 i}
\end{gathered}
$$

can be obtained.

In general, the $m \ll n$, so that the computational effort to solve Eq. (16) is little.

\subsection{Further analysis}

Once the basis vectors $\Phi_{m}$ have been calculated, the following procedure can be used to evaluate the eigenvalues and eigenvectors $\left(\phi_{i}, \lambda_{i}\right)$ for the modified structure:

1) To form modified matrices $K_{m}, M_{m}$ by Eqs. (10) and (11), and to introduce starting vectors $\Phi_{m}$ expressed by Eq. (13)

2) To factorize the stiffness matrix $K_{m}$

$$
K_{m}=L D L^{T}
$$

3) To solve the static problems to obtain the improved Ritz basis vectors

$$
K_{m} \bar{X}=M_{m} \Phi_{m}
$$

4) To perform the Rayleigh-Ritz analysis

$$
\begin{aligned}
& K_{R}=\bar{X}^{T} K_{m} \bar{X} \\
& M_{R}=\bar{X}^{T} M_{m} \bar{X} \\
& K_{R} Q_{R}=M_{R} Q_{R} \Omega_{R}^{2}
\end{aligned}
$$

5) To find approximate eigenvalues and eigenvectors by

$$
\Lambda=\Omega_{R}^{2}
$$

and

$$
\Phi+\bar{X} Q_{R}
$$

As shown later in numerical examples, satisfactory approximation results of the modified structure can not be got only with $\Phi_{m}$ directly as Ritz basis vectors. If during the first iteration of the subspace good approximation results of the modified structure can be obtained, it is unnecessary to perform more iterations.

\section{Numerical examples}

In order to illustrate the validity of the proposed method, two numerical examples are given.

\subsection{Plate structure}

Consider the initial design of a rectangle plate structure drawn with thin lines, shown in Fig. 1, with its parameters: the thickness of plate is $t=0.01 \mathrm{~m}$, the length and width of plate are $1 \mathrm{~m}$ and $0.3 \mathrm{~m}$ respectively. Fixed on both sides, the plate is discretisized into 44 nodes and 30 square plate members.

From the initial modal analysis, the first six eigenvalues $\Lambda_{0}$ are as follows:

$$
\begin{aligned}
& \lambda_{01}=0.118 \times 10^{6}, \lambda_{02}=0.556 \times 10^{6} \\
& \lambda_{03}=0.899 \times 10^{6}, \lambda_{04}=0.261 \times 10^{7} \\
& \lambda_{05}=0.351 \times 10^{7}, \lambda_{06}=0.724 \times 10^{7}
\end{aligned}
$$

Consider topological modifications of the added new nodes and members with thick lines, shown in Fig. 1. The changes of the stiffness matrix $\Delta K$ and mass matrix $\Delta M$ are formed by a finite element program.

For the purpose of comparison, the modal analysis of the modified structure are computed through the following four methods: 1) exact method (by subspace iteration), 2) direct method (by the initial eigenvectors $\Phi_{0}$ in Eq. (18) as the starting vectors directly), 3) Ritz method (by the expand basis vectors $\Phi_{m}$ as Ritz basis to perform the Ritz analysis), 4) the present method. The first three eigenvalues resulting from these four methods are shown in Table 1.

As seen in Table 1, the Ritz method results in wrong eigenvalues. Because the eigenvectors of the modified structure are similar to those of the initial structure, both the direct method and present method can give good approximate eigenvalues and eigenvectors.

\subsection{Truss structure}

Consider a truss structure (shown in Fig. 2) with its parameters: elasticity modulus $=2.1 \times 10^{11} \mathrm{~Pa}$, cross section area of each rod: $1 \times 10^{-3} \mathrm{~m}^{2}$, mass density: $7.8 \times 10^{3} \mathrm{~kg} / \mathrm{m}^{3}$. The length between node 2 and node 16 is $2.5 \mathrm{~m}$; between node 2 and node 3 is $0.5 \mathrm{~m}$; and the height of the structure is $0.4 \mathrm{~m}$. The truss structure is fixed at node 1, 3, 22 and 23.

From the initial modal analysis, the first six initial eigenvalues $\Lambda_{0}$ are:

$$
\begin{aligned}
& \lambda_{01}=0.580 \times 10^{7}, \lambda_{02}=0.120 \times 10^{8} \\
& \lambda_{03}=0.189 \times 10^{8}, \lambda_{04}=0.375 \times 10^{8} \\
& \lambda_{05}=0.469 \times 10^{8}, \lambda_{06}=0.791 \times 10^{8}
\end{aligned}
$$

The topological modifications with the added new 12 nodes 18 to 29 and 48 members are shown in Fig. 3 . The 


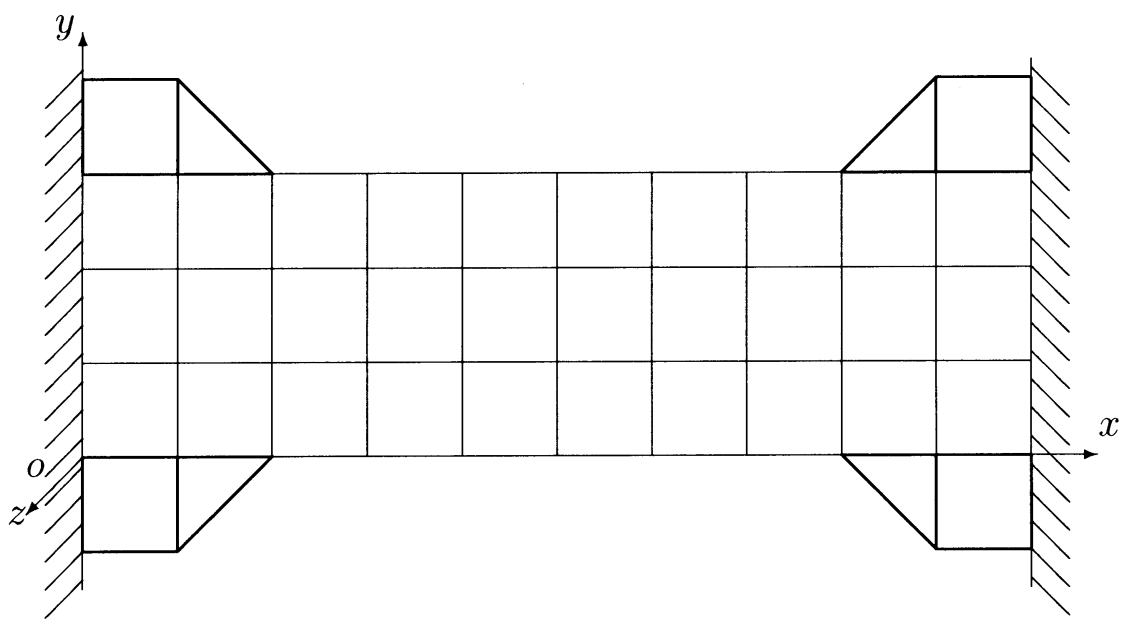

Fig. 1. Topological modifications of plate structure.

Table 1

The eigenvalues of modified structure

\begin{tabular}{cccccccc}
\hline Modes & Exact & Direct & error & Ritz & error & Present & error \\
\hline 1 & $0.1509 \mathrm{E} 6$ & $0.1557 \mathrm{E} 6$ & $3.16 \%$ & $0.1378 \mathrm{E} 7$ & $813.1 \%$ & $0.1557 \mathrm{E} 6$ & $3.14 \%$ \\
2 & $0.7326 \mathrm{E} 6$ & $0.7591 \mathrm{E} 6$ & $3.62 \%$ & $0.7049 \mathrm{E} 7$ & $862.1 \%$ & $0.7575 \mathrm{E} 6$ & $3.38 \%$ \\
3 & $0.1036 \mathrm{E} 7$ & $0.1111 \mathrm{E} 7$ & $7.25 \%$ & $0.1092 \mathrm{E} 8$ & $954.2 \%$ & $0.1111 \mathrm{E} 7$ & $7.21 \%$ \\
\hline
\end{tabular}

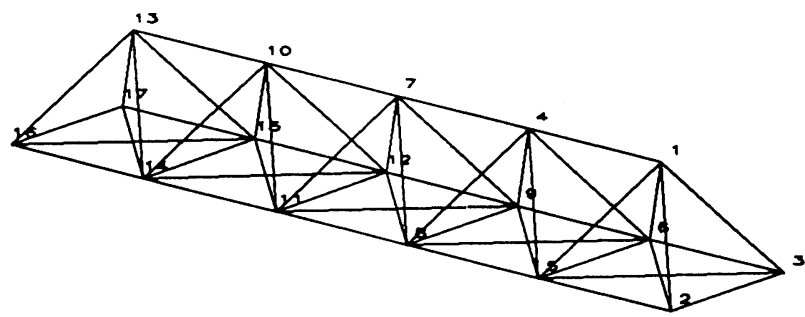

Fig. 2. Initial design of truss structure.

Table 2

The frequencies of modified structure $(\mathrm{Hz})$

\begin{tabular}{cccccccc}
\hline Modes & Exact & Direct & error & Ritz & error & Present & error \\
\hline 1 & 160.289 & 170.334 & $6.27 \%$ & 402.743 & $151.7 \%$ & 160.428 & $0.09 \%$ \\
2 & 237.456 & 296.462 & $24.85 \%$ & 545.219 & $129.6 \%$ & 237.678 & $0.09 \%$ \\
3 & 294.561 & 396.457 & $34.59 \%$ & 584.554 & $98.45 \%$ & 296.066 & $0.51 \%$ \\
\hline
\end{tabular}

changes of the stiffness matrix $\Delta K$ and mass matrix $\Delta M$ are formed by the finite element program. The $\Phi_{m}$ is given by Eqs. (13) and (16).

The initial eigenvectors $\Phi_{0}$ can not be used directly as the starting vectors in Eq. (18), because they do not include the information of the new added nodes. If the eigenvectors of the modified structure have distinct difference from the eigenvectors of the initial structure, they may result in wrong eigenvectors. For the purpose of comparison, the modal analysis of the modified structure are computed through the following four methods: 1) exact method, 2) direct method, 3) Ritz method and 4) the present method. The first three eigenvalues resulting from these four methods are shown in Table 2.

As seen in Table 2, the present method can obtain good approximate eigenvalues while the Ritz method results in wrong results. The corresponding first two mode shape vectors are shown in Figs 4 and 5, respectively. The present method can also give good approx- 


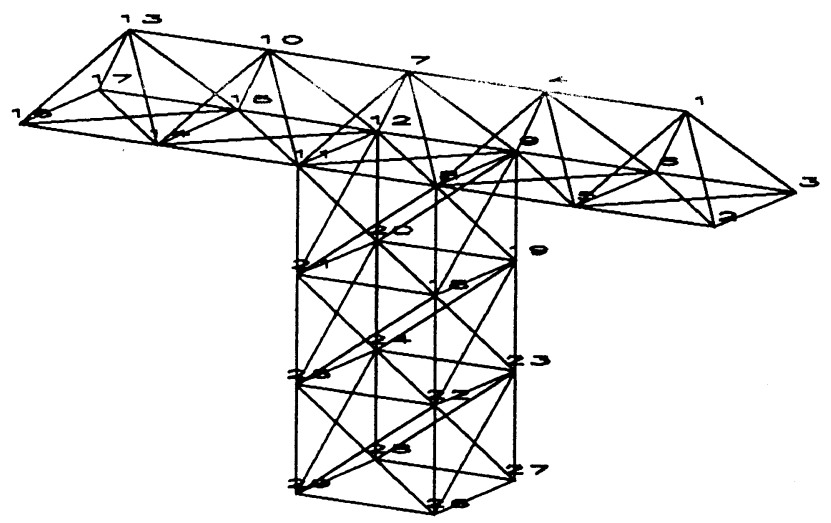

Fig. 3. Topological modifications of truss structure.

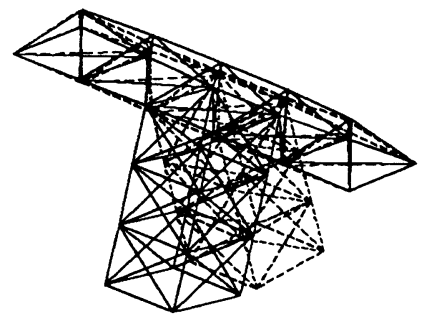

(a) Exact

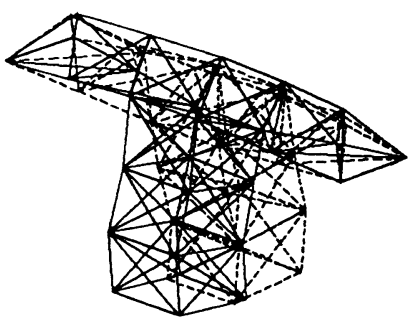

(b) Direct

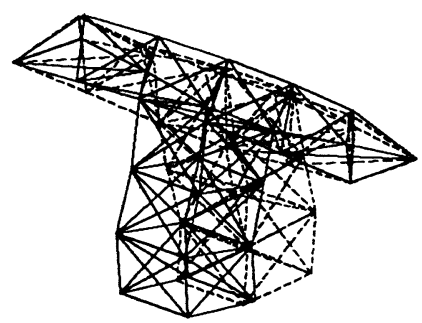

(c) Present

Fig. 4. First mode shape vectors of modified structure.

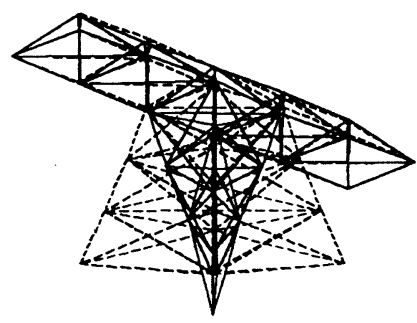

(a) Exact

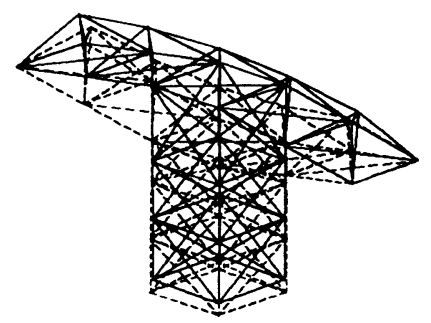

(b) Direct

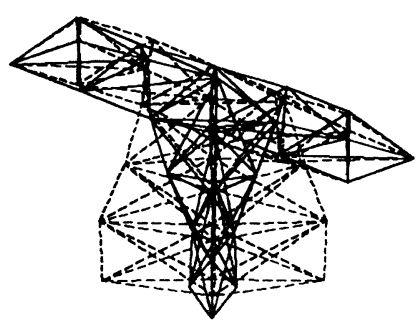

(c) Present

Fig. 5. Second mode shape vectors of modified structure.

imate mode shape vectors, and the second mode obtained by the direct method is not only inaccurate, but also misleading.

\section{Concluding remarks}

In this paper, a new modal reanalysis method for topological modifications of general finite element sys- tems has been presented. This presentation focuses on the most challenging case of addition of joints, in which the structural model and the number of DOF are changed. A simple reanalysis are first carried out to expand the basis vectors, so that the new DOF are included in the analysis model. The method does not require to reassemble the whole stiffness matrix and mass matrix of the modified structure, which only needs to assemble the changes of stiffness matrix and mass ma- 
trix for the added new nodes and members. Because the method deals with the stiffness matrix and mass matrix directly, it can be applied to general finite element systems.

With the numerical examples, the efficient and highquality approximate solutions in topological modifications can be obtained by the present method.

\section{Acknowledgement}

This work is supported by the National Natural Science Foundation of P.R. China and Mechanical Technology Development Foundation of P.R. China.

\section{References}

[1] Adelmen, H.M., and Haftka, R.T., Sensitivity analysis of discrete structural systems, AIAA Journal 24 (1986), 823-832.

[2] Chen, S.H., Matrix Perturbation Theory in Structural Dynam- ics, International Academic Publishers, 1993.

[3] Chen, S.H., Huang, C. and Liu, Z.S., Structural approximate reanalysis for topological modifications of finite element systems, AIAA J. 36 (1998), 1760-1762.

[4] Chen, T.Y., Structural modification with frequency response constraints for undamped MDOF systems, Computers \& Structures 36 (1990), 1013-1018.

[5] Cox, H.L., The Design of Structures of Least Weight, Pergamon, London, 1956.

[6] Haug, E.J., Komkov, V. and Choi, K.K., Design sensitivity analysis of structural systems, Academic Press, Orlando Fl, 1985.

[7] Kirsch, U. and Liu, S.H., Structural reanalysis for general layout modifications, AIAA J. 35 (1997), 382-388.

[8] Liang, P., Chen, S.H. and Huang, C., Moor-Penrose inverse method of topological variation of finite element systems, Computers \& Structures 62 (1997), 243-251.

[9] Liu, Z.S. and Chen, S.H., Reanalysis of static response and its design sensitivity of locally modified structures, Communication in Numerical Methods in Engineering 8 (1992), 797-800.

[10] Noor, A.K., Recent advances and applications of reduction methods, Applied Mechanics Reviews 47 (1994), 125-143.

[11] Topping, B.H.V., Shape optimization of skeletal structures: A Review, J Struct. Eng. ASCE 109 (1983), 1933-1951. 

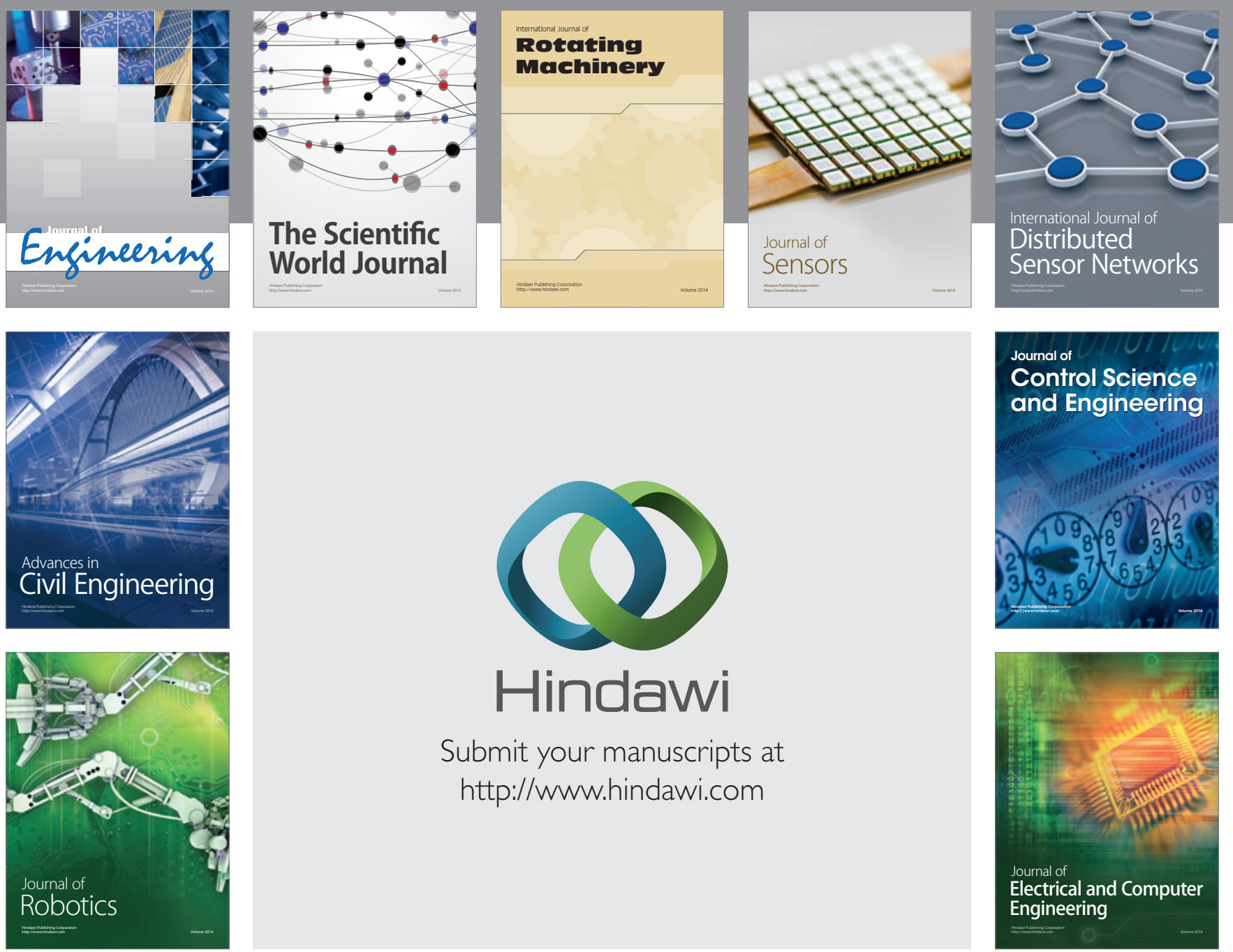

Submit your manuscripts at

http://www.hindawi.com
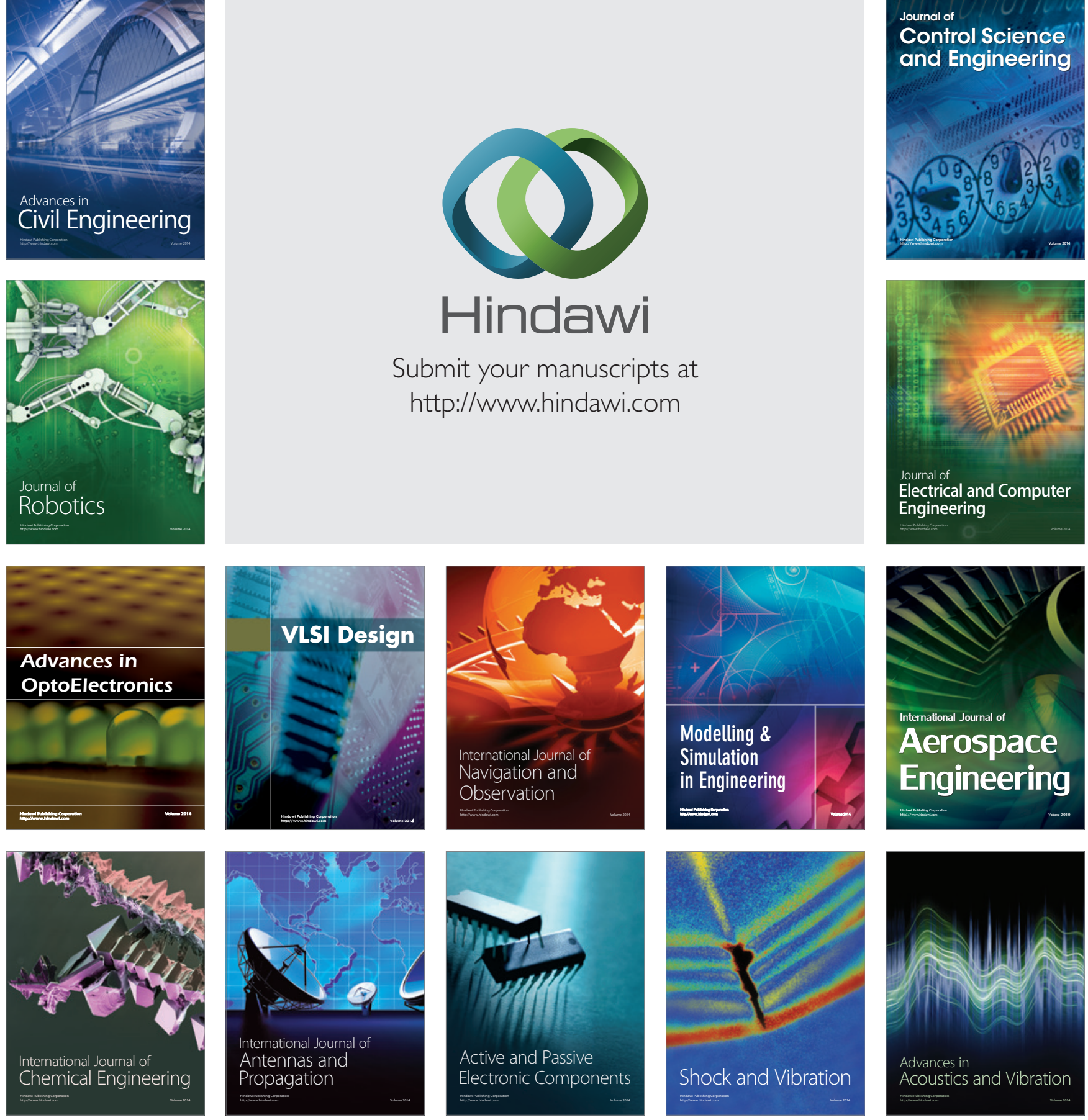